arXiv:1204.2679

\title{
FAPT: a Mathematica package for calculations in QCD Fractional Analytic Perturbation Theory
}

\author{
Alexander P. Bakulev ${ }^{\mathrm{a}}$, Vyacheslav L. Khandramai ${ }^{\mathrm{a}, \mathrm{b}}$ \\ ${ }^{a}$ Bogoliubov Laboratory of Theoretical Physics, JINR, 141980 Dubna, Russia \\ ${ }^{b}$ Gomel State Technical University, 246746 Gomel, Belarus
}

\begin{abstract}
We provide here all the procedures in Mathematica which are needed for the computation of the analytic images of the strong coupling constant powers in Minkowski $\left(\overline{\mathfrak{A}}_{\nu}\left(s ; n_{f}\right)\right.$ and $\left.\mathfrak{A}_{\nu}^{\text {glob }}(s)\right)$ and Euclidean $\left(\overline{\mathcal{A}}_{\nu}\left(Q^{2} ; n_{f}\right)\right.$ and $\left.\mathcal{A}_{\nu}^{\text {glob }}\left(Q^{2}\right)\right)$ domains at arbitrary energy scales ( $s$ and $Q^{2}$, correspondingly) for both schemes - with fixed number of active flavours $n_{f}=3,4,5,6$ and the global one with taking into account all heavy-quark thresholds. These singularity-free couplings are inevitable elements of Analytic Perturbation Theory (APT) in QCD [1-3], and its generalization - Fractional APT [4-6], needed to apply the APT imperative for renormalization-group improved hadronic observables.

PACS numbers: 12.38.Bx, 11.15.Bt, 11.10.Hi

Keywords: Analyticity, Fractional Analytic Perturbation Theory, Perturbative QCD, Renormalization group evolution
\end{abstract}

Email addresses: bakulev@theor.jinr.ru (Alexander P. Bakulev), v.khandramai@gmail.com (Vyacheslav L. Khandramai) 


\section{Program Summary}

Title of program: FAPT

Available from:

http://theor.jinr.ru/ bakulev/fapt.mat/FAPT.m

http://theor.jinr.ru/ bakulev/fapt.mat/FAPT_Interp.m

Computer for which the program is designed and others on which it is operable: Any work-station or PC where Mathematica is running.

Operating system or monitor under which the program has been tested: Windows $\mathrm{XP}$, Mathematica (versions 5 and 7).

No. of bytes in distributed program including test data etc.:

$47 \mathrm{kB}$ (main module FAPT.m) and $4 \mathrm{kB}$ (interpolation module FAPT_Interp.m);

$21 \mathrm{kB}$ (notebook FAPT_Interp.nb showing how to use the interpolation module);

$10888 \mathrm{kB}$ (interpolation data files: AcalGlob $\ell$ i. dat and UcalGlob $\ell$ i. dat with $\ell=$ $1,2,3,3 \mathrm{P}$, and 4$)^{1}$

\section{Distribution format: ASCII}

Nature of physical problem: The values of analytic images $\overline{\mathcal{A}}_{\nu}\left(Q^{2}\right)$ and $\overline{\mathfrak{A}}_{\nu}(s)$ of the QCD running coupling powers $\alpha_{\mathrm{s}}^{\nu}\left(Q^{2}\right)$ in Euclidean and Minkowski regions, correspondingly, are determined through the spectral representation in the QCD Analytic Perturbation Theory (APT). In the program FAPT we collect all relevant formulas and various procedures which allow for a convenient evaluation of $\overline{\mathcal{A}}_{\nu}\left(Q^{2}\right)$ and $\overline{\mathfrak{A}}_{\nu}(s)$ using numerical integrations of the relevant spectral densities.

Method of solution: FAPT uses Mathematica functions to calculate different spectral densities and then performs numerical integration of these spectral integrals to obtain analytic images of different objects.

Restrictions on the complexity of the problem: It could be that for an unphysical choice of the input parameters the results are out of any meaning.

Typical running time: For all operations the running time does not exceed a few seconds. Usually numerical integration is not fast, so that we advice to use arrays of precalculated data and apply then the routine Interpolate (as shown in supplied example of the program usage, namely in the notebook FAPT_Interp.nb).

\footnotetext{
${ }^{1}$ The notebook FAPT_Interp.nb and all interpolation data files are available from the same place in the form of the zipped archive FAPT_Interp.zip of the size $1844 \mathrm{kB}$. In order that Mathematica notebook FAPT_Interp.nb can use these precalculated data files one should place the directory . \sources $\backslash$ with all data files in the same directory as the main file FAPT_Interp.nb.
} 


\section{Introduction}

QCD perturbation theory $(\mathrm{PT})$ in the region of spacelike four-momentum transfer $\left(Q^{2}=-q^{2}>0\right.$ - hereafter we call it the Euclidean region) is based on expansions in a series over the powers of effective charge (or running coupling constant) $\alpha_{s}\left(Q^{2}\right)$, which in the one-loop approximation is given by $\alpha_{s}^{(1)}\left(Q^{2}\right)=\left(4 \pi / b_{0}\right) / L$ with $b_{0}$ being the first coefficient of the QCD beta function, Eq. (2)-(3), $L=\ln \left(Q^{2} / \Lambda^{2}\right)$, and $\Lambda=\Lambda_{\mathrm{QCD}}$ is the QCD scale parameter. The one-loop solution $\alpha_{s}^{(1)}\left(Q^{2}\right)$ has a pole singularity at $L=0$ called the Landau pole. The $\ell$-loop solution $\alpha_{s}^{(\ell)}\left(Q^{2}\right)$ of the renormalization group equation (2) has an $\ell$-root singularity of the type $L^{-1 / \ell}$ at $L=0$, which produces the pole as well in the $\ell$-order term $d_{\ell} \alpha_{s}^{\ell}\left(Q^{2}\right)$. This prevents the application of perturbative QCD in the low-momentum spacelike regime, $Q^{2} \sim \Lambda^{2}$, with the effect that hadronic quantities, calculated at the partonic level in terms of a power-series expansion in the running coupling, are not everywhere well defined.

Such a singularity appeared first in QED [7, 8] and was named "ghost" due to the negative residue at the corresponding propagator pole. It was interpreted as an indication that quantum field theory is self-contradictory. However, as was shown in [9, 10, it is only a hint about the PT inapplicability in the region where the expansion parameter is not small. Appearance of such "ghost" singularities from a theoretical point of view contradicts the causality principle in quantum field theory [10, 11], since it makes the Källen-Lehmann spectral representation impossible. It also complicates the determination of the effective charge in the timelike region $\left(q^{2}>0\right.$ - hereafter we call it the Minkowski region). In a seminal paper by N. N. Bogoliubov et al. of 1959 [12], the ghost-free effective coupling for QED has been constructed using the dispersion relation technique.

After the very appearance of QCD many researchers tried to determine the QCD effective charge in the Minkowski region, which is suitable for describing the processes of $e^{+} e^{-}$ annihilation into hadrons, as well as quarkonium and $\tau$-lepton decays into hadrons. Many such attempts used analytic continuation of the effective charge from the deep Euclidean region, in which perturbative QCD is known to work well, into a Minkowski one, where actual experiments were performed: $\alpha_{s}\left(Q^{2}\right) \rightarrow \alpha_{s}\left(s=-Q^{2}\right)$. In 1982 Radyushkin [13] and Krasnikov and Pivovarov [14] using the dispersion technique of [12] suggested regular (for $s \geq \Lambda^{2}$ ) QCD running coupling in Minkowski region, the well-known $\pi^{-1} \arctan (\pi / L)$.

In 1995 Jones and Solovtsov using variational approach [15] constructed the effective couplings in Euclidean and Minkowski domains which appears to be finite for all $Q^{2}$ and $s$ and satisfy analyticity integral conditions. Just in the same time Shirkov and Solovtsov [1, using the dispersion approach of [12, discovered ghost-free coupling $\mathcal{A}_{1}\left(Q^{2}\right)$, Eq. (25a), in Euclidean region and ghost-free coupling $\mathfrak{A}_{1}(s)$, Eq. (25b), in Minkowski region, which satisfy analyticity integral conditions:

$$
\mathcal{A}_{1}\left(Q^{2}\right)=Q^{2} \int_{0}^{\infty} \frac{\mathfrak{A}_{1}(\sigma)}{\left(\sigma+Q^{2}\right)^{2}} d \sigma ; \quad \mathfrak{A}_{1}(s)=\frac{1}{2 \pi i} \int_{-s-i \varepsilon}^{-s+i \varepsilon} \frac{\mathcal{A}_{1}(\sigma)}{\sigma} d \sigma .
$$

At the one-loop approximation the last coupling coincides with the Radyushkin one for 
$s \geq \Lambda^{2}$. This way of making the QCD's effective charge analytic in the timelike region was rediscovered later within an approach of fermion bubble resummation by Beneke and Braun [16], and also by Ball, Beneke, and Braun [17]. Due to the absence of singularities in these couplings, Shirkov and Solovtsov suggested to use this systematic approach, called Analytic Perturbation Theory (APT), for all $Q^{2}$ and $s$.

Recently the analytic and numerical methods, necessary to perform calculations in two- and three-loop approximations, were developed [18 24]. This approach was applied to the calculation of properties of a number of hadronic processes, including the width of inclusive $\tau$ lepton decay to hadrons [25 29], the scheme and renormalization-scale dependencies in the Bjorken [30, 31] and Gross-Llewellyn Smith [32] sum rules, the width of $\Upsilon$ meson decay to hadrons [33], etc. Moreover, APT was applied to the analysis of the processes with two scales rather than just a single scale, namely: the pion-photon transition form factor [34, 35] and the pion electromagnetic form factor in the $O\left(\alpha_{s}\right)$ order [34-36]. To summarize, we can say that APT (see reviews [37-39]) yields a sensible description of hadronic quantities in $\mathrm{QCD}$, though there are alternative approaches to the singularity of effective charge in QCD - in particular, with respect to the deep infrared region $Q^{2}<\Lambda^{2}$, where appearance of nonzero hadronic masses may be important [40 42]. The main advantage of the APT analysis is much more faster convergence of the APT non-power series as compared with the standard PT power series, see in [43, 44].

Three-point functions, used in describing the pion electromagnetic form factor or $\gamma^{*} \gamma \rightarrow \pi^{0}$ transition form factor, contain logarithmic contributions at the next-to-leading order of the QCD PT, related to the factorization scale. If one set the factorization scale proportional to the squared momentum-transfer, $\mu_{\mathrm{F}}^{2}=Q^{2}$, then these logarithms will go to zero, but additional RG factors of the type $\left(\alpha_{s}\left(Q^{2}\right) / \alpha_{s}\left(\mu_{0}^{2}\right)\right)^{\nu}$, with $\nu=\gamma_{n} /\left(2_{0}\right)$ being a fractional number, will appear in the Gegenbauer coefficients of the pion distribution amplitude. In both cases spectral densities, used to construct analytic images of hadronic amplitudes, should change. This observation led Karanikas and Stefanis [45, 46] to propose the concept of analytization "as a whole", meaning that one should construct analytic images not only of effective charge and its powers, but of the whole QCD amplitude under consideration.

A QCD inspired generalization of APT to the fractional powers of effective charge, called Fractional Analytic Perturbation Theory (FAPT), was done in [4, 6] (for a recent review see [47], for a recent generalization see [48]), followed by the application [5] to the analysis of the factorizable contribution to the pion electromagnetic form factor. The crucial advantage of FAPT in this case is that the perturbative results start to be less dependent on the factorization scale. This reminds the results, obtained with the APT, applied to the analysis of the pion form factor in the $O\left(\alpha_{s}^{2}\right)$ approximation, where the results also almost cease to depend on the choice of the renormalization scheme and its scale (for a detailed review see [47] and references therein). The process of the Higgs boson decay into a $b \bar{b}$ pair of quarks was studied within a framework of FAPT in the Minkowski region at the one-loop level in [49] and at the three-loop level- in [6]. Results on the resummation of non-power-series expansions of the Adler function of a scalar, $D_{S}$, and a vector, $D_{V}$, correlators within FAPT were presented in [50]. The interplay between 
higher orders of the perturbative QCD (pQCD) expansion and higher-twist contributions in the analysis of recent Jefferson Lab data on the lowest moment of the spin-dependent proton structure function, $\Gamma_{1}^{p}\left(Q^{2}\right)$, was studied in [51] using both standard QCD PT and (F)APT. FAPT technique was also applied to the analysis of the structure function $F_{2}(x)$ behavior at small values of $x$ [52, 53]. All these successful applications of (F)APT necessitate to have a reliable mathematical tool for calculations of spectral densities and analytic couplings which are implemented in FAPT ${ }^{2}$

In this paper we collect all relevant formulas which are necessary for the running of $\overline{\mathcal{A}}_{\nu}[L]$ and $\overline{\mathfrak{A}}_{\nu}[L]$ in the framework of APT and its fractional generalization, FAPT. We discuss their proper usage and provide easy-to-use Mathematica [56] procedures collected in the package FAPT. A few examples are given. Here we do not consider the inclusion of analytic images of logarithms multiplied by fractional powers of couplings, namely, $\left[\alpha_{s}\left(Q^{2}\right)\right]^{\nu} \cdot\left[\ln \left(Q^{2} / \Lambda^{2}\right)\right]^{m}$, which are needed for the full implementation of FAPT, - we postpone it to the next paper.

The outline of the paper is as follows. In the next Section we present the main formulas of perturbative QCD which are needed for the running of the strong coupling constant up to the four-loop level. Section 3 contains the basic formulas of APT and FAPT 33 Finally, in Section 4 we describe the most important procedures of the package FAPT and provide an example of using this package to produce some numerical estimations. We hope that for most practical applications it should be sufficient. In the Appendix we supply the complete collection of the developed procedures.

\section{Basics of the QCD running coupling}

The running of the coupling constant of QCD, $\alpha_{\mathrm{s}}\left(\mu^{2}\right)=\alpha_{\mathrm{s}}[L]$ with $L=\ln \left[\mu^{2} / \Lambda^{2}\right]$, is defined throught

$$
\frac{d \alpha_{\mathrm{s}}[L]}{d L}=\beta\left(\alpha_{\mathrm{s}}[L] ; n_{f}\right)=-\alpha_{\mathrm{s}}[L] \sum_{k \geq 0} b_{k}\left(n_{f}\right)\left(\frac{\alpha_{\mathrm{s}}[L]}{4 \pi}\right)^{k+1},
$$

\footnotetext{
${ }^{2}$ This task has been partially realized for both APT and its massive generalization [42] as the Maple package QCDMAPT in [54] and as the Fortran package QCDMAPT_F in [55]. Both these realizations are limited to the case of fixed number of active quarks $N_{f}=3$ only, and use approximate expressions for the two- and higher-loop perturbative couplings, compare, for example, Eq. (33) in 54 and our Eq. (7).

${ }^{3}$ Note here that FAPT includes APT as a partial case for the integer values of indices.

${ }^{4}$ We use notations $f\left(Q^{2}\right)$ and $f[L]$ in order to specify the arguments we mean - squared momentum $Q^{2}$ or its $\operatorname{logarithm} L=\ln \left(Q^{2} / \Lambda^{2}\right)$, that is $f[L]=f\left(\Lambda^{2} \cdot e^{L}\right)$ and $\Lambda^{2}$ is usually referred to $n_{f}=3$ region.
} 
where $n_{f}$ is the number of active flavours. The coefficients are given by [57 66]

$$
\begin{aligned}
b_{0}\left(n_{f}\right)= & 11-\frac{2}{3} n_{f}, \\
b_{1}\left(n_{f}\right)= & 102-\frac{38}{3} n_{f}, \\
b_{2}\left(n_{f}\right)= & \frac{2857}{2}-\frac{5033}{18} n_{f}+\frac{325}{54} n_{f}^{2}, \\
b_{3}\left(n_{f}\right)= & \frac{149753}{6}+3564 \zeta_{3}-\left[\frac{1078361}{162}+\frac{6508}{27} \zeta_{3}\right] n_{f} \\
& +\left[\frac{50065}{162}+\frac{6472}{81} \zeta_{3}\right] n_{f}^{2}+\frac{1093}{729} n_{f}^{3} .
\end{aligned}
$$

$\zeta$ is Riemann's zeta function, with values $\zeta_{2}=\pi^{2} / 6$ and $\zeta_{3} \approx 1.202057$. It is convenient to introduce the following notations:

$$
\beta_{f} \equiv \frac{b_{0}\left(n_{f}\right)}{4 \pi}, \quad a\left(\mu^{2} ; n_{f}\right) \equiv \beta_{f} \alpha_{\mathbf{s}}\left(\mu^{2} ; n_{f}\right) \quad \text { and } \quad c_{k}\left(n_{f}\right) \equiv \frac{b_{k}\left(n_{f}\right)}{b_{0}\left(n_{f}\right)^{k+1}} .
$$

Then Eq. (2) in the $l$-loop approximation can be rewritten in the following form:

$$
\frac{d a_{(\ell)}\left[L ; n_{f}\right]}{d L}=-\left(a_{(\ell)}\left[L ; n_{f}\right]\right)^{2}\left[1+\sum_{k \geq 1}^{\ell} c_{k}\left(n_{f}\right)\left(a_{(\ell)}\left[L ; n_{f}\right]\right)^{k}\right] .
$$

In the one-loop $(l=1)$ approximation $\left(c_{k}\left(n_{f}\right)=b_{k}\left(n_{f}\right)=0\right.$ for all $\left.k \geq 1\right)$ we have a solution

$$
a_{(1)}[L]=\frac{1}{L}
$$

with the Landau pole singularity at $L \rightarrow 0$. In the two-loop $(l=2)$ approximation $\left(c_{k}\left(n_{f}\right)=b_{k}\left(n_{f}\right)=0\right.$ for all $\left.k \geq 2\right)$ the exact solution of Eq. (2) is also known [67, 68]

$$
a_{(2)}\left[L ; n_{f}\right]=\frac{-c_{1}^{-1}\left(n_{f}\right)}{1+W_{-1}\left(z_{W}[L]\right)} \quad \text { with } \quad z_{W}[L]=-c_{1}^{-1}\left(n_{f}\right) e^{-1-L / c_{1}\left(n_{f}\right)},
$$

where $W_{-1}[z]$ is the appropriate branch of Lambert function.

The three- and higher-loop solutions $a_{(\ell)}\left[L ; n_{f}\right]$ can be expanded in powers of the two-loop one, $a_{(2)}\left[L ; n_{f}\right]$, as has been suggested in [19, 22, 24, 29]:

$$
a_{(\ell)}\left[L ; n_{f}\right]=\sum_{n \geq 1} C_{n}^{(\ell)}\left(a_{(2)}\left[L ; n_{f}\right]\right)^{n} .
$$

Coefficients $C_{n}^{(\ell)}$ are known and can be evaluated recursively. We use in our routine for the three-loop coupling expansion up to the 9-th power included:

$$
\begin{gathered}
C_{1}^{(3)}=1, \quad C_{2}^{(3)}=0, \quad C_{3}^{(3)}=c_{2}, \quad C_{4}^{(3)}=0, \quad C_{5}^{(3)}=\frac{5}{3} c_{2}^{2}, \quad C_{6}^{(3)}=\frac{-1}{12} c_{1} c_{2}^{2}, \\
C_{7}^{(3)}=\frac{1}{20} c_{1}^{2} c_{2}^{2}+\frac{16}{5} c_{2}^{3}, \quad C_{8}^{(3)}=\frac{-1}{30} c_{1}^{3} c_{2}^{2}-\frac{23}{60} c_{1} c_{2}^{3} \\
C_{9}^{(3)}=\frac{1}{42} c_{1}^{4} c_{2}^{2}+\frac{103}{420} c_{1}^{2} c_{2}^{3}+\frac{2069}{315} c_{2}^{4} .
\end{gathered}
$$



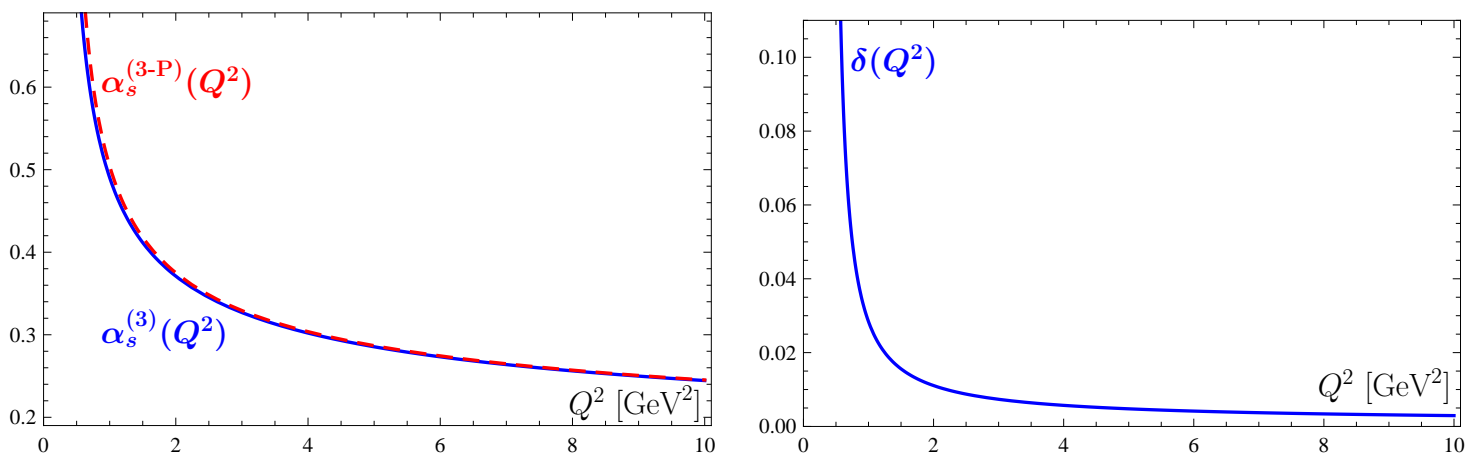

Figure 1: Left panel: Comparison of the standard three-loop coupling $\alpha_{s}^{(3)}\left(Q^{2}\right)$ (solid blue line) with the three-loop Pade one $\alpha_{s}^{(3 \mathrm{P})}\left(Q^{2}\right)$ (dashed red line). Right panel: Relative accuracy $\delta\left(Q^{2}\right)=\left(\alpha_{s}^{(3 \mathrm{P})}\left(Q^{2}\right)-\right.$ $\left.\alpha_{s}^{(3)}\left(Q^{2}\right)\right) / \alpha_{s}^{(3)}\left(Q^{2}\right)$ of the three-loop Pade coupling as compared with the standard three-loop one.

As has been shown in [24] this expansion has a finite radius of convergence, which appears to be sufficiently large for all values of $n_{f}$ of practical interest. Note here that this method of expressing the higher- $\ell$-loop coupling in powers of the two-loop one is equivalent to the 't Hooft scheme, where one put by hands all coefficients in $\beta$-function, except $b_{0}$ and $b_{1}$, equal to zero and effectively takes into account all higher coefficients $b_{i}$ by redefining perturbative coefficients $d_{i}$ (see for more detail in [69]).

Another possibility for obtaining the "exact" three-loop solution is provided by the so-called Pade approximation scheme. It is based on the Pade-type modification of the three-loop beta function:

$$
\begin{aligned}
& \beta_{(3 \mathrm{P})}\left(\alpha_{\mathrm{s}}\right)=-\frac{\alpha_{\mathrm{s}}^{2}}{4 \pi}\left[b_{0}+\frac{b_{1} \alpha_{\mathrm{s}} /(4 \pi)}{1-b_{2} \alpha_{\mathrm{s}} /\left(4 \pi b_{1}\right)}\right], \\
& \frac{d a_{(3 \mathrm{P})}[L]}{d L}=-a_{(3 \mathrm{P})}^{2}[L]\left[1+\frac{c_{1} a_{(3 \mathrm{P})}[L]}{1-c_{2} a_{(3 \mathrm{P})}[L] / c_{1}}\right] .
\end{aligned}
$$

The last equation can be solved exactly with the help of the same Lambert function (here the explicit dependence on $n_{f}$ is not shown for shortness):

$$
a_{(3 \mathrm{P})}[L]=\frac{-c_{1}^{-1}}{1-c_{2} / c_{1}^{2}+W_{-1}\left(z_{W}^{(3 \mathrm{P})}[L]\right)} \quad \text { with } z_{W}^{(3 \mathrm{P})}[L]=-c_{1}^{-1} e^{-1+c_{2} / c_{1}^{2}-L / c_{1}}
$$

The relative accuracy of this solution as compared with numerical solution of the standard three-loop equation (5) with $l=3$ is better than $1 \%$ for $Q^{2} \geq 2 \mathrm{GeV}^{2}$ (with $\Lambda_{3}^{(3)}=$ $356 \mathrm{MeV}$ ) and better than $0.5 \%$ for $Q^{2} \geq 5 \mathrm{GeV}^{2}$, cf. Fig. 1 .

In the four-loop approximation we use the same Eq. (8) with corresponding coefficients

$$
C_{n}^{(4)}=C_{n}^{(3)}+\Delta_{n}^{(4)}
$$



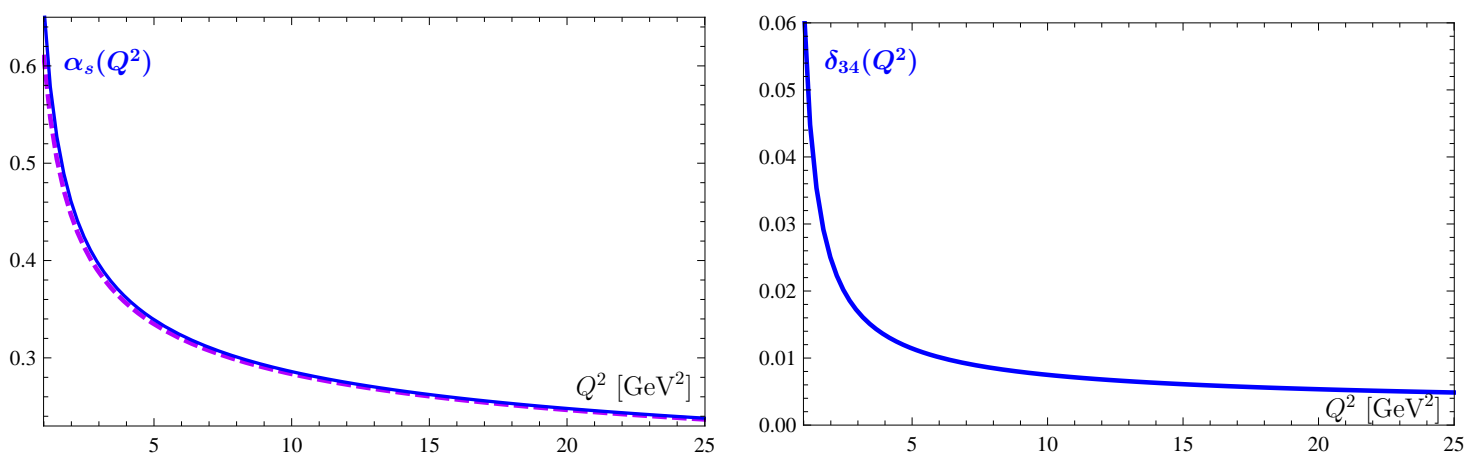

Figure 2: Left panel: Comparison of the four-loop coupling $\alpha_{s}^{(4)}\left(Q^{2}\right)$ (solid blue line) with the three-loop one $\alpha_{s}^{(3)}\left(Q^{2}\right)$ (dashed violet line). Right panel: Relative accuracy $\delta_{34}\left(Q^{2}\right)=\left(\alpha_{s}^{(4)}\left(Q^{2}\right)-\right.$ $\left.\alpha_{s}^{(3)}\left(Q^{2}\right)\right) / \alpha_{s}^{(4)}\left(Q^{2}\right)$ of the three-loop coupling as compared with the four-loop one.

and

$$
\begin{gathered}
\Delta_{1}^{(4)}=\Delta_{2}^{(4)}=\Delta_{3}^{(4)}=0, \quad \Delta_{4}^{(4)}=c_{3}, \quad \Delta_{5}^{(4)}=\frac{-c_{1} c_{3}}{6}, \quad \Delta_{6}^{(4)}=\frac{c_{1}^{2} c_{3}}{12}+2 c_{2} c_{3}, \\
\Delta_{7}^{(4)}=\frac{-c_{1}^{3} c_{3}}{20}-\frac{4 c_{1} c_{2} c_{3}}{5}+\frac{11 c_{3}^{2}}{20}, \quad \Delta_{8}^{(4)}=\frac{c_{1}^{4} c_{3}}{30}+\frac{9 c_{1}^{2} c_{2} c_{3}}{20}+\frac{19 c_{2}^{2} c_{3}}{3}-\frac{49 c_{1} c_{3}^{2}}{120}, \\
\Delta_{9}^{(4)}=\frac{c_{1}^{5} c_{3}}{42}-\frac{41 c_{1}^{3} c_{2} c_{3}}{140}-\frac{946 c_{1} c_{2}^{2} c_{3}}{315}+\frac{134 c_{2} c_{3}^{2}}{35}+\frac{149 c_{1}^{2} c_{3}^{2}}{504} .
\end{gathered}
$$

In the left panel of Fig. 2 we show both couplings, the four-loop $\alpha_{s}^{(4)}\left(Q^{2}\right)$ (solid blue line), and the three-loop $\alpha_{s}^{(3)}\left(Q^{2}\right)$ (dashed violet line) with fixed number of active flavors $n_{f}=4$. We normalize both couplings to the same value $\alpha_{s}\left(m_{Z}^{2}\right)=0.119$ at the $Z$-boson mass scale. Numerically, as can be seen in the right panel of Fig. 2, the relative deviation $\delta_{34}\left(Q^{2}\right)=\left(\alpha_{s}^{(4)}\left(Q^{2}\right)-\alpha_{s}^{(3)}\left(Q^{2}\right)\right) / \alpha_{s}^{(4)}\left(Q^{2}\right)$ varies from $6 \%$ at $Q^{2}=1 \mathrm{GeV}^{2}$ to $0.5 \%$ at $Q^{2}=25 \mathrm{GeV}^{2}$. We also compared the four-loop coupling, calculated in accord with Eq. (8), with coupling, calculated using package RunDec [70] with the same normalization $\alpha_{s}\left(m_{Z}^{2}\right)=0.119$, - the relative deviation appears to vary from $0.2 \%$ at $Q^{2}=1 \mathrm{GeV}^{2}$ to $0.04 \%$ at $Q^{2}=25 \mathrm{GeV}^{2}$.

\subsection{Global scheme}

Here we consider the scheme of the so-called "global pQCD" in which the heavy-quark thresholds are taken into account. We follow here to Shirkov-Solovtsov approach [1, 18, 20] with the following values of pole masses of $c, b$, and $t$ quarks: $m_{c}=1.65 \mathrm{GeV}, m_{b}=$ $4.75 \mathrm{GeV}$ and $m_{t}=172.5 \mathrm{GeV}$. In the $\overline{\mathrm{MS}}$ scheme of the standard pQCD one needs to match the running coupling values in Euclidean domain at $Q^{2}$ corresponding to these masses: $M_{4}=m_{c}, M_{5}=m_{b}$ and $M_{6}=m_{t}$. In order to implement these matching conditions we need to use the original QCD coupling

$$
\alpha_{\mathrm{s}}^{(\ell)}\left(Q^{2} ; n_{f}\right)=\frac{4 \pi}{b_{0}\left(n_{f}\right)} a^{(\ell)}\left(Q^{2} ; n_{f}\right)
$$


where the indicator ${ }^{(\ell)}$ signals about the loop order of the approximation we use ${ }^{5}$

In what follows we use all logarithms $L$ with respect to three-flavor scale $\Lambda_{3}^{2}$ :

$$
L\left(Q^{2}\right)=\ln \left(Q^{2} / \Lambda_{3}^{2}\right) .
$$

Recalculation to all other scales is realized with the help of finite additions:

$$
\ln \left(Q^{2} / \Lambda_{k}^{2}\right)=L\left(Q^{2}\right)+\lambda_{k} \quad \text { with } \quad \lambda_{k} \equiv \ln \left(\Lambda_{3}^{2} / \Lambda_{k}^{2}\right)
$$

and $\Lambda_{k}$ - the corresponding to the specified value $n_{f}=k$ scale of QCD. We also define the corresponding logarithmic values at the thresholds $M_{k}(k=4 \div 6)$ :

$$
L_{k}\left(\Lambda_{3}\right) \equiv \ln \left(M_{k}^{2} / \Lambda_{3}^{2}\right)
$$

All QCD scales $\Lambda_{f}, f=4,5,6$, we treat as functions of the single parameter, namely, the three-flavor scale $\Lambda_{3}$ :

$$
\Lambda_{f} \rightarrow \Lambda_{f}\left(\Lambda_{3}\right) \quad \text { with } \quad \Lambda_{3}>\Lambda_{4}\left(\Lambda_{3}\right)>\Lambda_{5}\left(\Lambda_{3}\right)>\Lambda_{6}\left(\Lambda_{3}\right)
$$

which should be defined from matching conditions for the running coupling at the heavyquark thresholds.

For an illustration we consider here the two-loop approximation with the running coupling $\alpha_{\mathrm{s}}^{(2)}\left[L ; n_{f}\right]$

$$
\alpha_{\mathrm{s}}^{(2)}\left[L ; n_{f}\right]=\frac{-4 \pi}{b_{0}\left(n_{f}\right) c_{1}\left(n_{f}\right)\left[1+W_{-1}\left(z_{W}\left[L ; n_{f}\right]\right)\right]}
$$

with $z_{W}\left[L ; n_{f}\right]=\left(1 / c_{1}\left(n_{f}\right)\right) \exp \left[-1+i \pi-L / c_{1}\left(n_{f}\right)\right]$. Then matching conditions are

$$
\begin{aligned}
\alpha_{\mathrm{s}}^{(2)}\left[L_{4}\left(\Lambda_{3}\right) ; 3\right] & =\alpha_{\mathrm{s}}^{(2)}\left[L_{4}\left(\Lambda_{3}\right)+\lambda_{4} ; 4\right] ; \\
\alpha_{\mathrm{s}}^{(2)}\left[L_{5}\left(\Lambda_{3}\right)+\lambda_{4} ; 4\right] & =\alpha_{\mathrm{s}}^{(2)}\left[L_{5}\left(\Lambda_{3}\right)+\lambda_{5} ; 5\right] ; \\
\alpha_{\mathrm{s}}^{(2)}\left[L_{6}\left(\Lambda_{3}\right)+\lambda_{5} ; 5\right] & =\alpha_{\mathrm{s}}^{(2)}\left[L_{6}\left(\Lambda_{3}\right)+\lambda_{6} ; 6\right] .
\end{aligned}
$$

These relations define constants $\lambda_{k}$ with $k=4 \div 6$ as functions of variable $\Lambda_{3}$, namely

$$
\lambda_{k} \rightarrow \lambda_{k}^{(2)}\left(\Lambda_{3}\right)
$$

and, as a consequence, the continuous global effective QCD coupling

$$
\begin{aligned}
\alpha_{\mathrm{s}}^{\text {glob; }(2)}\left(Q^{2}, \Lambda_{3}\right) & =\alpha_{\mathrm{s}}^{(2)}\left[L\left(Q^{2}\right) ; 3\right] \theta\left(Q^{2}<M_{4}^{2}\right) \\
& +\alpha_{\mathrm{s}}^{(2)}\left[L\left(Q^{2}\right)+\lambda_{4}^{(2)}\left(\Lambda_{3}\right) ; 4\right] \theta\left(M_{4}^{2} \leq Q^{2}<M_{5}^{2}\right) \\
& +\alpha_{\mathrm{s}}^{(2)}\left[L\left(Q^{2}\right)+\lambda_{5}^{(2)}\left(\Lambda_{3}\right) ; 5\right] \theta\left(M_{5}^{2} \leq Q^{2}<M_{6}^{2}\right) \\
& +\alpha_{\mathrm{s}}^{(2)}\left[L\left(Q^{2}\right)+\lambda_{6}^{(2)}\left(\Lambda_{3}\right) ; 6\right] \theta\left(M_{6}^{2} \leq Q^{2}\right) .
\end{aligned}
$$

\footnotetext{
${ }^{5}$ Note here that the dependence $a^{(\ell)}\left(Q^{2} ; n_{f}\right)$ on $n_{f}$ is the consequence of Eq. (5), where for $l>1$ one has $n_{f}$-dependent coefficients $c_{k}\left(n_{f}\right)$.
} 
Here is the list of partial values of $\Lambda_{f}^{(2)}\left(\Lambda_{3}\right), \lambda_{f}^{(2)}\left(\Lambda_{3}\right)$ and $L_{f}\left(\Lambda_{3}\right)$ with $f=4,5,6$ for $\Lambda_{3}=400 \mathrm{MeV}$ :

$$
\begin{aligned}
\Lambda_{4}^{(2)} & =333 \mathrm{MeV}, & \Lambda_{5}^{(2)} & =233 \mathrm{MeV}, & \Lambda_{6}^{(2)} & =98 \mathrm{MeV} \\
\lambda_{4}^{(2)} & =0.367, & \lambda_{5}^{(2)} & =1.08, & \lambda_{6}^{(2)} & =2.82 ; \\
L_{4} & =2.197, & L_{5} & =4.750, & L_{6} & =12.162 .
\end{aligned}
$$

In our m-file we use the following realizations. The QCD scales are encoded as $\Lambda 1\left[\Lambda, n_{f}\right], \Lambda 2\left[\Lambda, n_{f}\right]$, and $\Lambda 3\left[\Lambda, n_{f}\right]$ (in Mathematica capital Greek symbol $\Lambda$ can be written as $\backslash[$ CapitalLambda]):

$$
\backslash[\text { CapitalLambda }] \ell[\Lambda, k]=\Lambda \ell\left[\Lambda, n_{f}=k\right]=\Lambda_{k}^{(\ell)}(\Lambda),(\ell=1 \div 4,3 \mathrm{P} ; k=4 \div 6) \text {, }
$$

the threshold logarithms - as $\lambda \ell 4[\Lambda], \lambda \ell 5[\Lambda]$, and $\lambda \ell 6[\Lambda]$ (in Mathematica Greek symbol $\lambda$ can be written as $\backslash[$ Lambda] ):

$$
\backslash[\text { Lambda }] \ell k[\Lambda]=\lambda \ell k[\Lambda]=\ln \left(\Lambda^{2} / \Lambda \ell[\Lambda, k]^{2}\right), \quad(\ell=1 \div 4,3 \mathrm{P} ; k=4 \div 6),
$$

the running QCD couplings with fixed $n_{f}$ - as $\alpha \operatorname{Bar} 1\left[Q^{2}, n_{f}, \Lambda\right], \alpha \operatorname{Bar} 2\left[Q^{2}, n_{f}, \Lambda\right]$, and $\alpha \operatorname{Bar} 3\left[Q^{2}, n_{f}, \Lambda\right]$ (in Mathematica Greek symbol $\alpha$ can be written as $\backslash[\mathrm{Alpha}]$ ):

$$
\backslash[\text { Alpha }] \operatorname{Bar} \ell\left[Q^{2}, n_{f}, \Lambda\right]=\alpha \operatorname{Bar} \ell\left[Q^{2}, n_{f}, \Lambda\right]=\alpha_{\mathrm{s}}^{(\ell)}\left[\ln \left(Q^{2} / \Lambda^{2}\right) ; n_{f}\right],(\ell=1 \div 4,3 \mathrm{P}),
$$

and the global running QCD couplings — as $\alpha$ Glob1 $\left[Q^{2}, \Lambda\right], \quad \alpha \operatorname{Glob} 2\left[Q^{2}, \Lambda\right]$, and $\alpha \operatorname{Glob} 3\left[Q^{2}, \Lambda\right]$ :

$$
\backslash[\mathrm{Alpha}] \text { Glob } \ell\left[Q^{2}, \Lambda\right]=\alpha \operatorname{Glob} \ell\left[Q^{2}, \Lambda\right]=\alpha_{\mathrm{s}}^{\text {glob; }(\ell)}\left(Q^{2}, \Lambda\right),(\ell=1 \div 4,3 \mathrm{P}),
$$

To be more specific, we consider here an example. We assume that the two-loop $\alpha_{\mathrm{s}}$ is given at the $Z$-boson scale as $\alpha_{\mathrm{s}}^{(2)}\left[\ln \left(m_{Z}^{2} / \Lambda^{2}\right) ; 5\right]=0.119$. We want to evaluate the corresponding values of the QCD scales $\Lambda_{3}, \Lambda_{4}$, and $\Lambda_{5}$ and the coupling $\alpha_{\mathrm{s}}^{\text {glob; }(\ell)}\left(Q^{2}, \Lambda\right)$ at the scale $Q=M_{5}$. We show a possible Mathematica realization of this task.

$\operatorname{In}[1]:=<<$ FAPT.m;

Comment: NumDefFAPT is a set of Mathematica rules in our package which assigns typical values to the physical parameters used in our procedures.

$\operatorname{In}[2]:=\{M Z=$ MZboson/.NumDefFAPT,$M b=M Q 5 / . N u m D e f F A P T\}$

Out $[2]=\{91.19,4.75\}$

Comment: evaluation of $\mathrm{L} 23=\Lambda_{3}^{(2)}$ from $\alpha_{\mathrm{s}}^{(2)}\left[\ln \left(m_{Z}^{2} / \Lambda^{2}\right) ; 5\right]$ based on the explicit solution, Eq. (18), Eq. (21).

$\operatorname{In}[3]:=\mathrm{L} 23=1 \mathrm{x} /$. FindRoot $[\backslash[\mathrm{Alpha}] \mathrm{Glob} 2[\mathrm{MZ} \sim 2,1 \mathrm{x}]==0.119,\{1 \mathrm{x}, 0.1,0.3\}]$ Out $[3]=0.387282$ 
Comment: evaluation of $\mathrm{L} 24=\Lambda_{4}^{(2)}$ and $\mathrm{L} 25=\Lambda_{5}^{(2)}$ from $\mathrm{L} 23=\Lambda_{3}^{(2)}$ based on Eq. 23a).

In $[4]:=\{$ L24= $\backslash[$ CapitalLambda $] 2[\mathrm{~L} 23,4]$, L25=\[CapitalLambda $] 2[\mathrm{~L} 23,5]\}$

Out $[4]=\{0.321298,0.224033\}$

Comment: evaluation of $\alpha_{\mathrm{s}}^{\text {glob;(2) }}\left(M_{b}^{2}\right)$ from $\mathrm{L} 23=\Lambda_{3}^{(2)}$.

In $[5]:=\backslash[\mathrm{Alpha}] \mathrm{Glob} 2[\mathrm{Mb} \leadsto 2, \mathrm{~L} 23]$

Out [5] $=0.218894$

\section{Basics of FAPT}

In the end of the previous section we used for the running QCD couplings with fixed $n_{f}$ the Bar notations - $\alpha \operatorname{Bar} 1\left[Q^{2}, n_{f}, \Lambda\right], \alpha \operatorname{Bar} 2\left[Q^{2}, n_{f}, \Lambda\right]$, and $\alpha \operatorname{Bar} 3\left[Q^{2}, n_{f}, \Lambda\right]$. We did it on purpose to have a direct connection to our previous papers on the subject [4, 6, 47], where we used the normalized coupling $a\left(\mu^{2}\right)=\beta_{f} \alpha_{\mathrm{s}}\left(\mu^{2}\right)$, cf. Eq. (4). To be in line with these definitions, we also introduce analogous expressions for the fixed- $N_{f}$ quantities with standard normalization, i.e.,

$$
\overline{\mathcal{A}}_{\nu}\left(Q^{2}\right)=\frac{\mathcal{A}_{\nu}\left(Q^{2}\right)}{\beta_{f}^{\nu}}, \quad \overline{\mathfrak{A}}_{\nu}(s)=\frac{\mathfrak{A}_{\nu}(s)}{\beta_{f}^{\nu}},
$$

which correspond to the analytic couplings $\mathcal{A}_{\nu}$ and $\mathfrak{A}_{\nu}$ in the Shirkov-Solovtsov terminology [1].

The basic objects in the (F)APT approach are spectral densities $\bar{\rho}_{\nu}^{(\ell)}\left(\sigma ; n_{f}\right)$ which enter the Källen-Lehmann spectral representation for the analytic couplings:

$$
\begin{aligned}
\overline{\mathcal{A}}_{\nu}^{(\ell)}\left[L ; n_{f}\right] & =\int_{0}^{\infty} \frac{\bar{\rho}_{\nu}^{(\ell)}\left(\sigma ; n_{f}\right)}{\sigma+Q^{2}} d \sigma=\int_{-\infty}^{\infty} \frac{\bar{\rho}_{\nu}^{(\ell)}\left[L_{\sigma} ; n_{f}\right]}{1+\exp \left(L-L_{\sigma}\right)} d L_{\sigma}, \\
\overline{\mathfrak{A}}_{\nu}^{(\ell)}\left[L_{s} ; n_{f}\right] & =\int_{s}^{\infty} \frac{\bar{\rho}_{\nu}^{(\ell)}\left(\sigma ; n_{f}\right)}{\sigma} d \sigma=\int_{L_{s}}^{\infty} \bar{\rho}_{\nu}^{(\ell)}\left[L_{\sigma} ; n_{f}\right] d L_{\sigma},
\end{aligned}
$$

It is convenient to use the following representation for spectral functions

$$
\bar{\rho}_{\nu}^{(\ell)}\left[L ; n_{f}\right]=\frac{1}{\pi} \operatorname{Im}\left(\alpha_{\mathrm{s}}^{(\ell)}\left[L-i \pi ; n_{f}\right]\right)^{\nu}=\frac{\sin \left[\nu \varphi_{(\ell)}\left[L ; n_{f}\right]\right]}{\pi\left(\beta_{f} R_{(\ell)}\left[L ; n_{f}\right]\right)^{\nu}},
$$

which is based on the module-phase representation of a complex number

$$
\alpha_{\mathrm{s}}^{(\ell)}\left[L-i \pi ; n_{f}\right]=\frac{a_{(\ell)}\left[L-i \pi ; n_{f}\right]}{\beta_{f}\left(n_{f}\right)}=\frac{e^{i \varphi_{(\ell)}\left[L ; n_{f}\right]}}{\beta_{f}\left(n_{f}\right) R_{(\ell)}\left[L ; n_{f}\right]} .
$$

In the one-loop approximation the corresponding functions have the most simple form

$$
\varphi_{(1)}[L]=\arccos \left(\frac{L}{\sqrt{L^{2}+\pi^{2}}}\right), \quad R_{(1)}[L]=\sqrt{L^{2}+\pi^{2}}
$$


and do not depend on $n_{f}$, whereas at the two-loop order they have a more complicated form

$$
\begin{aligned}
R_{(2)}\left[L ; n_{f}\right] & =c_{1}\left(n_{f}\right)\left|1+W_{1}\left(z_{W}\left[L-i \pi ; n_{f}\right]\right)\right| \\
\varphi_{(2)}\left[L ; n_{f}\right] & =\arccos \left[\operatorname{Re}\left(\frac{-R_{(2)}\left[L ; n_{f}\right]}{1+W_{1}\left(z_{W}\left[L-i \pi ; n_{f}\right]\right)}\right)\right]
\end{aligned}
$$

with $W_{1}[z]$ being the appropriate branch of Lambert function. In the three-loop approximation we use either Eq. (8) and then obtain

$$
\begin{aligned}
& R_{(3)}[L]=\left|\frac{e^{i \varphi_{(2)}[L]}}{R_{(2)}[L]}+\sum_{k \geq 3} C_{k}^{(3)} \frac{e^{i k \varphi_{(2)}[L]}}{R_{(2)}^{k}[L]}\right|^{-1} ; \\
& \varphi_{(3)}[L]=\arccos \left[\frac{R_{(3)}[L] \cos \left(\varphi_{(2)}[L]\right)}{R_{(2)}[L]}+\sum_{k \geq 3} C_{k}^{(3)} \frac{R_{(3)}[L] \cos \left(k \varphi_{(2)}[L]\right)}{R_{(2)}^{k}[L]}\right]
\end{aligned}
$$

or Eq. (11) — and then obtain

$$
\begin{aligned}
& R_{(3 \mathrm{P})}[L]=c_{1}\left|1-\frac{c_{2}}{c_{1}^{2}}+W_{1}\left(z_{W}^{(3 \mathrm{P})}[L-i \pi]\right)\right| \\
& \varphi_{(3 \mathrm{P})}[L]=\arccos \left[\boldsymbol{\operatorname { R e }}\left(\frac{-R_{(3 \mathrm{P})}[L]}{1-\left(c_{2} / c_{1}^{2}\right)+W_{1}\left(z_{W}^{(3 \mathrm{P})}[L-i \pi]\right)}\right)\right] .
\end{aligned}
$$

In the four-loop approximation we use Eq. (8) and then obtain

$$
\begin{aligned}
& R_{(4)}[L]=\left|\frac{e^{i \varphi_{(2)}[L]}}{R_{(2)}[L]}+\sum_{k \geq 3} C_{k}^{(4)} \frac{e^{i k \varphi_{(2)}[L]}}{R_{(2)}^{k}[L]}\right|^{-1} ; \\
& \varphi_{(4)}[L]=\arccos \left[\frac{R_{(4)}[L] \cos \left(\varphi_{(2)}[L]\right)}{R_{(2)}[L]}+\sum_{k \geq 3} C_{k}^{(4)} \frac{R_{(4)}[L] \cos \left(k \varphi_{(2)}[L]\right)}{R_{(2)}^{k}[L]}\right]
\end{aligned}
$$

Here we do not show explicitly the $n_{f}$ dependence of the corresponding quantities - it goes inside through $R_{(2)}[L]=R_{(2)}\left[L ; n_{f}\right], \varphi_{(2)}[L]=\varphi_{(2)}\left[L ; n_{f}\right], C_{k}^{(3)}=C_{k}^{(3)}\left[n_{f}\right], C_{k}^{(4)}=C_{k}^{(4)}\left[n_{f}\right]$, $c_{k}=c_{k}\left(n_{f}\right)$ with $k=1 \div 3$, and $z_{W}^{(3 \mathrm{P})}[L]=z_{W}^{(3 \mathrm{P})}\left[L ; n_{f}\right]$. In the left panel of Fig. 3 we show both spectral densities in comparison. On the right panel of this figure we show the relative deviation of the Pade spectral density from the standard one: one can see that it varies from $+1 \%$ at $L \approx-7$, reduces to $-2 \%$ at $L \approx 0$, and then reaches the maximum of $+2 \%$ at $L \approx 3.5$.

In accordance with Eq. (21) the global spectral densities are constructed through the $n_{f}$-fixed ones in the following manner:

$$
\begin{aligned}
\rho_{\nu}^{(\ell) ; \operatorname{glob}}\left[L_{\sigma}, \Lambda_{3}\right] & =\bar{\rho}_{\nu}^{(\ell)}\left[L_{\sigma} ; 3\right] \theta\left(L_{\sigma}<L_{4}\left(\Lambda_{3}\right)\right)+\bar{\rho}_{\nu}^{(\ell)}\left[L_{\sigma}+\lambda_{6}^{(\ell)}\left(\Lambda_{3}\right) ; 6\right] \theta\left(L_{6}\left(\Lambda_{3}\right) \leq L_{\sigma}\right) \\
& +\bar{\rho}_{\nu}^{(\ell)}\left[L_{\sigma}+\lambda_{4}^{(\ell)}\left(\Lambda_{3}\right) ; 4\right] \theta\left(L_{4}\left(\Lambda_{3}\right) \leq L_{\sigma}<L_{5}\left(\Lambda_{3}\right)\right) \\
& +\bar{\rho}_{\nu}^{(\ell)}\left[L_{\sigma}+\lambda_{5}^{(\ell)}\left(\Lambda_{3}\right) ; 5\right] \theta\left(L_{5}\left(\Lambda_{3}\right) \leq L_{\sigma}<L_{6}\left(\Lambda_{3}\right)\right)
\end{aligned}
$$



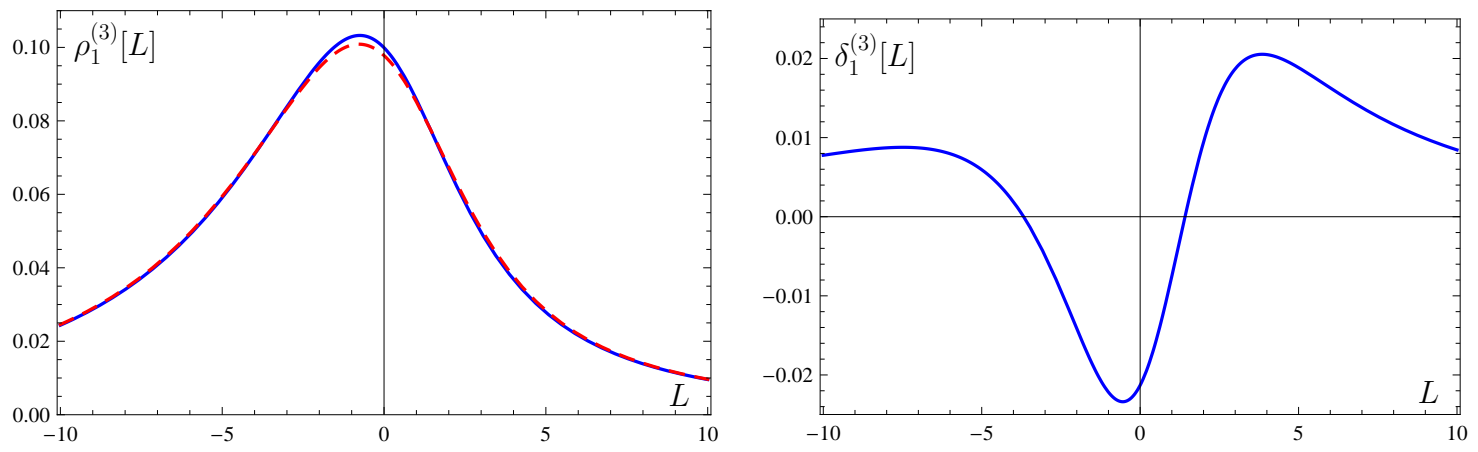

Figure 3: Left panel: Comparison of the standard three-loop spectral density $\rho_{1}^{(3)}[L]$ (solid blue line) with the three-loop Pade one $\rho_{1}^{(3 \mathrm{P})}[L]$ (dashed red line). Right panel: Relative accuracy $\delta_{1}^{(3)}[L]=$ $\left(\rho_{1}^{(3 \mathrm{P})}[L]-\rho_{1}^{(3)}[L]\right) / \rho_{1}^{(3)}[L]$ of the three-loop Pade spectral density as compared with the standard threeloop one.

with $L_{\sigma} \equiv \ln \left(\sigma / \Lambda_{3}^{2}\right)$ and the corresponding global analytic couplings are

$$
\begin{aligned}
\mathcal{A}_{\nu}^{(\ell) ; \operatorname{glob}}\left[L, \Lambda_{3}\right] & =\int_{-\infty}^{\infty} \frac{\rho_{\nu}^{(\ell) ; \operatorname{glob}}\left[L_{\sigma}, \Lambda_{3}\right]}{1+\exp \left(L-L_{\sigma}\right)} d L_{\sigma}, \\
\mathfrak{A}_{\nu}^{(\ell) ; \operatorname{glob}}\left[L, \Lambda_{3}\right] & =\int_{L}^{\infty} \rho_{\nu}^{(\ell) ; \operatorname{glob}}\left[L_{\sigma}, \Lambda_{3}\right] d L_{\sigma}
\end{aligned}
$$

\section{FAPT Procedures}

In our package FAPT.m we use the following realizations for the spectral densities. RhoBar $\ell\left[L, n_{f}, \nu\right]$ returns $\ell$-loop spectral density $\bar{\rho}_{\nu}^{(\ell)}(\ell=1,2,3,3 \mathrm{P}, 4)$ of fractional-power $\nu$ at $L=\ln \left(Q^{2} / \Lambda^{2}\right)$ and at fixed number of active quark flavors $n_{f}$ :

$$
\operatorname{RhoBar} \ell[L, k, \nu]=\bar{\rho}_{\nu}^{(\ell)}\left[L ; n_{f}=k\right], \quad(\ell=1 \div 4,3 \mathrm{P} ; k=3 \div 6),
$$

whereas RhoGlob $\ell\left[L, \nu, \Lambda_{3}\right]$ returns the global $\ell$-loop spectral density $\bar{\rho}_{\nu}^{(\ell) \text {;glob }}\left[L ; \Lambda_{3}\right](\ell=$ $1,2,3,3 \mathrm{P}, 4)$ of fractional-power $\nu$ at $L=\ln \left(Q^{2} / \Lambda_{3}^{2}\right)$, cf. and with $\Lambda_{3}$ being the QCD $n_{f}=3$-scale:

$$
\text { RhoGlob } \ell\left[L, \nu, \Lambda_{3}\right]=\bar{\rho}_{\nu}^{(\ell) ; \text { glob }}\left[L ; \Lambda_{3}\right], \quad(\ell=1 \div 4,3 \mathrm{P}),
$$

Analogously, AcalBar $\ell\left[L, n_{f}, \nu\right]$ returns $\ell$-loop $(\ell=1,2,3,3 \mathrm{P}, 4)$ analytic image of fractional-power $\nu$ coupling $\overline{\mathcal{A}}_{\nu}^{(\ell)}\left[L ; n_{f}\right]$ in Euclidean domain,

$$
\text { AcalBar } \ell[L, k, \nu]=\overline{\mathcal{A}}_{\nu}^{(\ell)}\left[L ; n_{f}=k\right], \quad(\ell=1 \div 4,3 \mathrm{P} ; k=3 \div 6),
$$

and UcalBar $\ell\left[L, n_{f}, \nu\right]$ returns $\ell$-loop $(\ell=1,2,3,3 \mathrm{P}, 4)$ analytic image of fractional-power $\nu$ coupling $\overline{\mathfrak{A}}_{\nu}^{(\ell)}\left[L, n_{f}\right]$ in Minkowski domain,

$$
\text { UcalBar } \ell[L, k, \nu]=\overline{\mathfrak{A}}_{\nu}^{(\ell)}\left[L ; n_{f}=k\right], \quad(\ell=1 \div 4,3 \mathrm{P} ; k=3 \div 6),
$$


In global case AcalGlob $\ell\left[L, \nu, \Lambda_{3}\right]$ returns $\ell$-loop analytic image of fractional-power $\nu$ coupling $\mathcal{A}_{\nu}^{(\ell) ; g l o b}\left[L, \Lambda_{3}\right]$ in Euclidean domain,

$$
\text { AcalGlob } \ell\left[L, \nu, \Lambda_{3}\right]=\mathcal{A}_{\nu}^{(\ell) ; g l o b}\left[L, \Lambda_{3}\right], \quad(\ell=1 \div 4,3 \mathrm{P}),
$$

and UcalGlob $\ell\left[L, \nu, \Lambda_{3}\right]$ returns $\ell$-loop analytic image of fractional-power $\nu$ coupling $\mathfrak{A}_{\nu}^{(\ell) ; g l o b}\left[L, \Lambda_{3}\right]$ in Minkowski domain,

$$
\text { UcalGlob } \ell\left[L, \nu, \Lambda_{3}\right]=\mathfrak{A}_{\nu}^{(\ell) ; g l o b}\left[L, \Lambda_{3}\right], \quad(\ell=1 \div 4,3 \mathrm{P}) .
$$

We consider here an example of using this quantities in case of Mathematica 7. We assume that the two-loop QCD scale $\Lambda_{3}$ is fixed at the value $\Lambda_{3}=0.387 \mathrm{GeV}$ defined at the end of section 2.1.

$\operatorname{In}[1]:=<<$ FAPT. $\mathrm{m}$;

$\operatorname{In}[2]:=\mathrm{L} 23=0.387$

We determine the value of the two-loop QCD scale L23APT $=\Lambda_{3}^{(2) \text {;APT }}$ in APT, corresponding to the same value 0.119 as before, but now for the global analytic coupling:

In $[3]:=$ MZ $=$ MZboson $/$. NumDefFAPT

Out $[3]=91.19$

In $[4]:=$ L23APT $=1 \mathrm{x} /$. FindRoot $[\mathrm{Aca}] \mathrm{Glob} 2\left[\log \left[\mathrm{MZ} ` 2 / 1 \mathrm{x}^{\wedge} 2\right], 1,1 \mathrm{x}\right]$

$==0.119,\{1 \mathrm{x}, 0.35,0.45\}]$;

Out $[4]=0.379788$

Now we evaluate the value of $\mathcal{A}_{\nu}^{(2) \text {; }{ }^{10 b}}[L, \mathrm{~L} 23 \mathrm{APT}]$ for $L=-5.0,-3.0,-1.0,1.0,3.0,5.0$ with indication of the needed time:

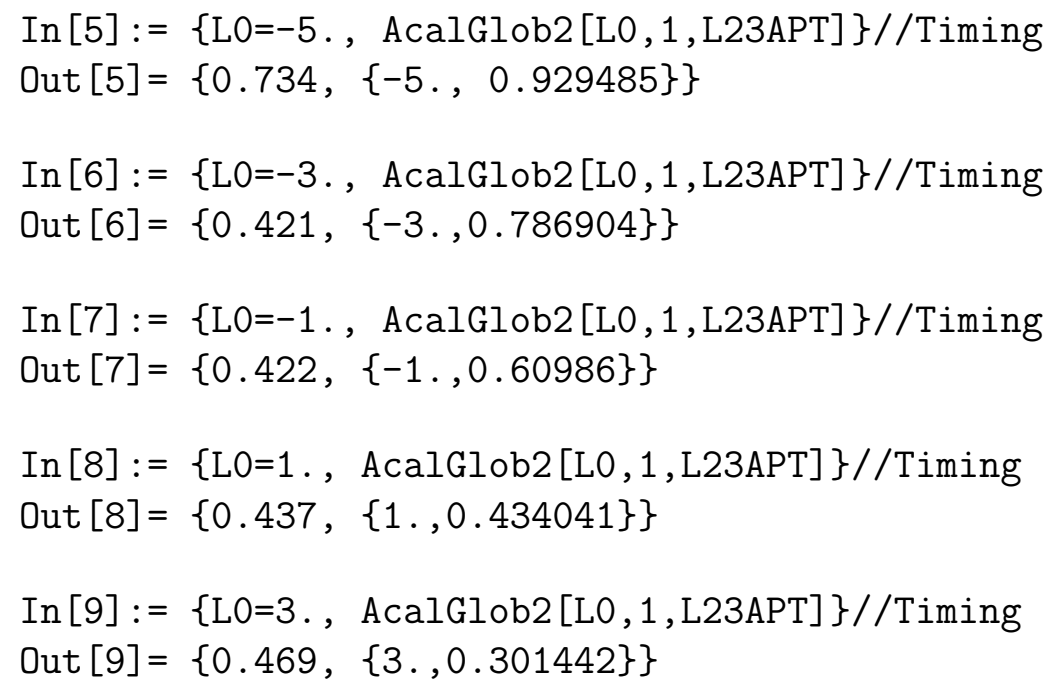



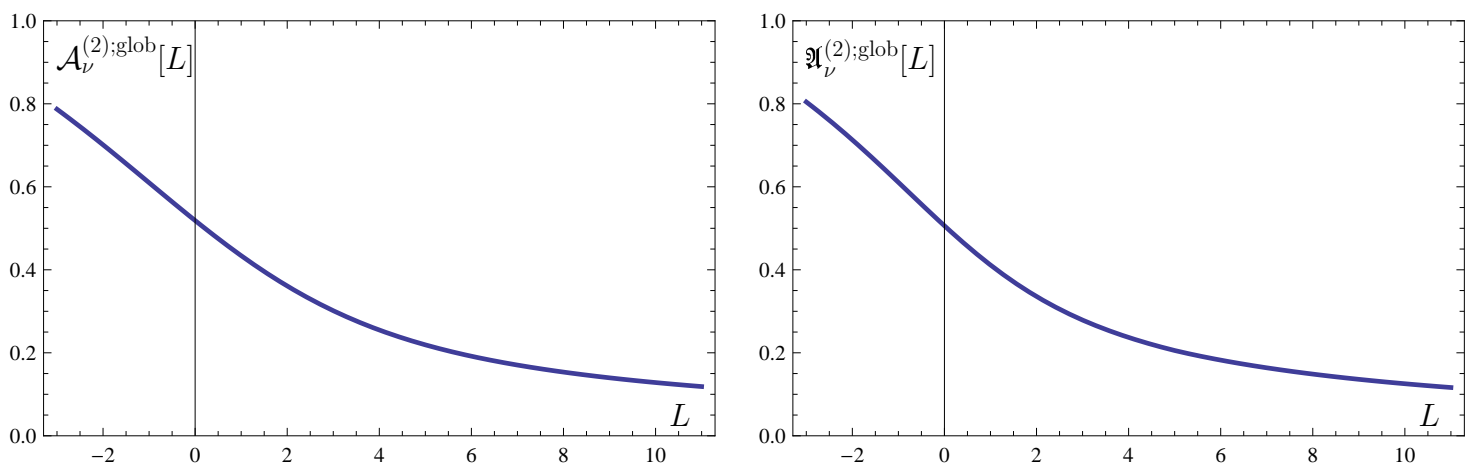

Figure 4: Left panel: Graphics produced in Out[10] for $\mathcal{A}_{\nu}^{(2) ; \text { glob }}[L, \mathrm{~L} 23 \mathrm{APT}]$ as a function of $L$. Right panel: Graphics produced in Out[11] for $\mathfrak{A}_{\nu}^{(2) ; \text { glob }}[L, \mathrm{~L} 23 \mathrm{APT}]$ as a function of $L$.

In $[10]:=\{\mathrm{LO}=5 ., \mathrm{AcalGlob} 2[\mathrm{LO}, 1, \mathrm{~L} 23 \mathrm{APT}]\} / /$ Timing

Out $[10]=\{0.531,\{5 ., 0.219137\}\}$

Now we create a two-dimensional plot of $\mathcal{A}_{\nu}^{(2) \text {;glob }}[L$, L23APT $]$ and $\mathfrak{A}_{\nu}^{(2) \text {;glob }}[L$, L23APT $]$ for $L \in[-3,11]$ with indication of the needed time:

In $[11]:=$ Plot $[$ AcalGlob2 [L , 1, L23APT],$\{\mathrm{L},-3,11\}$, MaxRecursion->1]//Timing

Out $[11]=\{19.843$, Graphics (see in the left panel of Fig. $\backslash, 4)\}$

In [12] := Plot [UcalGlob2 [L , 1, L23APT] , \{L , -3,11\}, MaxRecursion->1]//Timing

Out $[12]=\{14.656$, Graphics (see in the right panel of Fig. \,4) $\}$

\section{Interpolation}

The calculation of the spectral integrals is a computational task requiring a long time, especially if one is using the result in another numerical integration procedure. Therefore, it seems reasonable to pre-compute analytic images of couplings for a fixed set of argument values, consisting of $N$ points for each argument. For example we will consider in what follows the case of $\mathcal{A}_{\nu}^{(1) ; g l o b}\left[L, \nu, \Lambda_{3}^{(1)}\right]$. We will be interested in the following ranges of arguments: $L \in[-5,5], \Lambda_{3}^{(1)} \in[0.2,0.5]$, and $\nu=\in[0.5,1.5]$. Then

$$
\begin{aligned}
\operatorname{Lmin}=-5 ; & \operatorname{Lmax}=5 ; & \mathrm{DL}=(\operatorname{Lmax}-\operatorname{Lmin}) /(N-1) ; \\
\nu \min =0.5 ; & \nu \max =1.5 ; & \mathrm{D} \nu=(\nu \max -\nu \min ) /(N-1) ; \\
\Lambda \min =0.2 ; & \Lambda \max =0.5 ; & \mathrm{D} \Lambda=(\Lambda \max -\Lambda \min ) /(N-1) .
\end{aligned}
$$

The table of calculated values is generated by Mathematica using the following command

$$
\mathrm{DATA}=\mathrm{Flatten}\left[\mathrm{Table}\left[\left\{\left\{L_{i}, \nu_{j}, \Lambda_{k}\right\}, \operatorname{AcalGlob} 1\left[L_{i}, \nu_{j}, \Lambda_{k}\right]\right\},\{i, N\},\{j, N\},\{k, N\}\right], 2\right]
$$

where $L_{i}=\operatorname{Lmin}+(i-1) \mathrm{DL}, \nu_{j}=\nu \min +(j-1) \mathrm{D} \nu$, and $\Lambda_{k}=\Lambda \min +(k-1) \mathrm{D} \Lambda$. Then we save all calculated results in the file "AcalGlob1i.dat": 


\section{In [2] : $\quad X Y=N[D A T A] ;\{$ outFile = OpenWrite ["AcalGlob1i.dat"], Write[outFile, XY], Close[outFile]\} \\ Out [2]: \{OutputStream ["AcalGlob1i.dat", 15], Null, "AcalGlob1i.dat"\}}

After that we can read them and use interpolation to reproduce function $\mathcal{A}_{\nu}^{(1) ; g l o b}\left[L, \nu, \Lambda_{3}^{(1)}\right]$ in the considered ranges of arguments values:

\section{In [3] : $\quad$ DATA $=\operatorname{Read["AcalGlob1i.dat"];~}$$$
\text { AcalGlob1Interp = Interpolation [DATA] }
$$

in order to select the appropriate value of $N$. Now we can analyze the accuracy of interpolation. In Fig 5 we show the dependencies of interpolation errors on the number of the used points $N$. One can see, that using the interpolation at $N=6$ for $\mathcal{A}_{\nu}^{(\ell) ; g l o b}$ and $\mathfrak{A}_{\nu}^{(\ell) ; g l o b}$ provided accuracy not worse $0.005 \%$.

In the previous case we investigated the dependence of the accuracy of interpolation on the number of points at fixed $L, \nu$ and $\Lambda_{3}$. Let us now consider how the accuracy of


Figure 5: Relative errors of the interpolation procedure for $\mathcal{A}_{\nu}^{(\ell) ; g l o b}$ (left panel) and $\mathfrak{A}_{\nu}^{(\ell) ; g l o b}$ (right panel) calculated at various loop orders with fixed $L=3.5, \nu=1.1$ and $\Lambda_{3}=0.36 \mathrm{GeV}$.
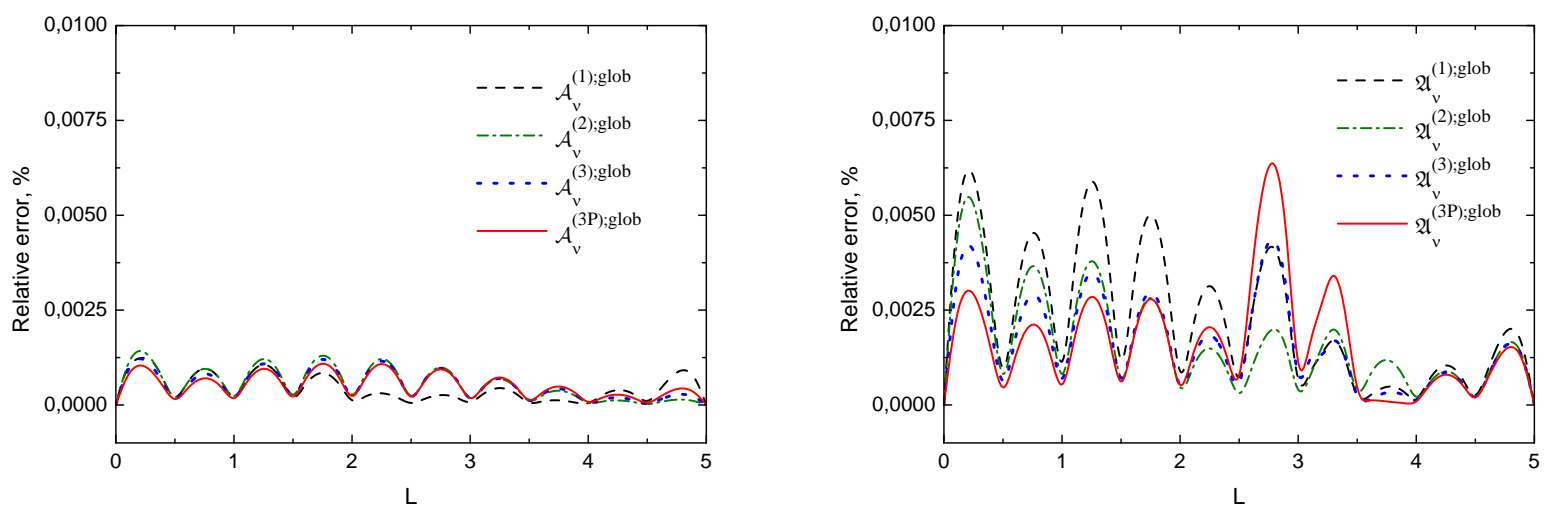

Figure 6: Relative error of the interpolation procedure for $\mathcal{A}_{\nu=1.1}^{g l o b}$ (left panel) and $\mathfrak{A}_{\nu=1.1}^{\text {glob }}$ (right panel), calculated at various loop orders with $\Lambda_{3}=0.36 \mathrm{GeV}$ for $N=11$ number of points. 
the interpolation depends on the $L$. These results are shown in Fig. 6. From the last figure one can see that the maximum error of interpolation corresponds to the region $L=0 \div 5$. The error in $\mathcal{A}_{\nu=0.6}^{(1) ; g l o b}$ is less than in $\mathfrak{A}_{\nu=1.1}^{(1) ; g l o b}$. In any case, using $N=11$ points for interpolation of pre-computed data for each parameter $L, \nu$ and $\Lambda_{3}$ provides an error less than $0.01 \%$.

To obtain the results much faster one can use module FAPT_Interp.m which consists of procedures AcalGlob $\ell\left[L, \nu, \Lambda_{3}\right]$ and UcalGlob $\ell\left[L, \nu, \Lambda_{3}\right]$. They are based on interpolation using the basis of the precalculated data in the ranges $L=[-5 ; 13] ; \nu^{1 \text {-loop }}=[0.5 ; 4.0]$ and $\Lambda_{n_{f}=3}^{1 \text {-loop }}=[0.150 ; 0.300] ; \nu^{2 \text {-loop }}=[0.5 ; 5.0]$ and $\Lambda_{n_{f}=3}^{\text {2-loop }}=[0.300 ; 0.450] ; \nu^{3 \text {-loop }}=[0.5 ; 6.0]$ and $\Lambda_{n_{f}=3}^{3 \text {-loop }}=[0.300 ; 0.450] ; \nu^{4 \text {-loop }}=[0.5 ; 7.0]$ and $\Lambda_{n_{f}=3}^{4 \text {-loop }}=[0.300 ; 0.450]$. For example, in the four-loop case module FAPT_Interp.m contains procedures

AcalGlob4i = Interpolation [Read[". \\sources\\AcalGlob4i.dat"]]; UcalGlob4i = Interpolation[Read[". \\sources\\UcalGlob4i.dat"]];

which should be used with the same arguments $L, \nu$, and $\Lambda_{3}$ as the original procedures AcalGlob $\ell\left[L, \nu, \Lambda_{3}\right]$ and UcalGlob $\ell\left[L, \nu, \Lambda_{3}\right]$. They provide much faster results of calculations with high enough accuracy:

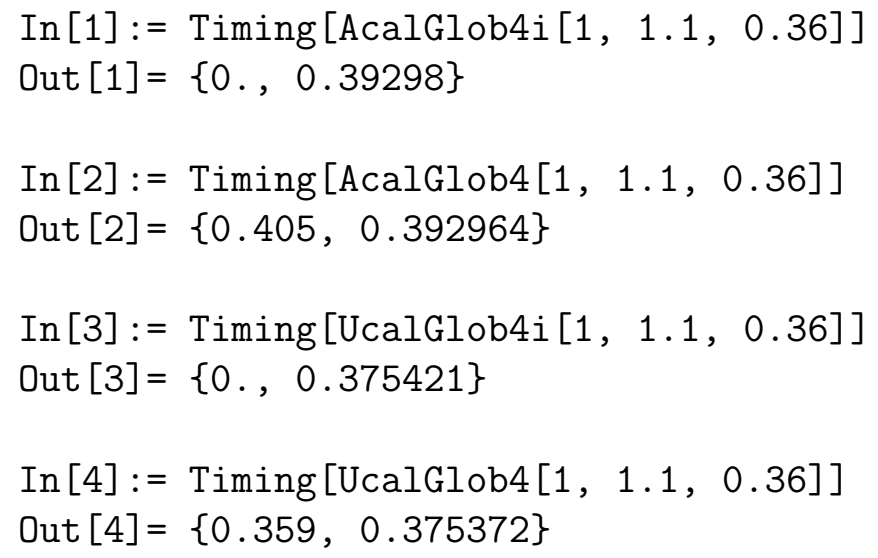

\section{Acknowledgments}

We would like to thank Andrei Kataev, Sergey Mikhailov, Irina Potapova, Dmitry Shirkov, and Nico Stefanis for stimulating discussions and useful remarks. This work was supported in part by the Russian Foundation for Fundamental Research (Grant No. 11-01-00182) and the BRFBR-JINR Cooperation Program under contract No. F10D-002.

\section{Appendix A. Numerical parameters}

Here we shortly describe numerical parameters used in the package. 
First, in FAPT.m we use the pole masses of heavy quarks and $Z$-boson, collected in the set NumDefFAPT:

$$
\begin{array}{llll}
\text { MQ4 : } & M_{c}=1.65 \mathrm{GeV}, & \text { MQ5 : } & M_{b}=4.75 \mathrm{GeV} ; \\
\text { MQ6 : } & M_{t}=172.5 \mathrm{GeV}, & \text { MZboson : } & M_{Z}=91.19 \mathrm{GeV} .
\end{array}
$$

Note here that all mass variables and parameters are measured in GeVs. That means, for example, that in all procedures of our package the following value MQ4 $=1.65$ is used. The package RunDec of [70] is using the set NumDef with slightly different values of these parameters $\left(M_{c}=1.6 \mathrm{GeV}, M_{b}=4.7 \mathrm{GeV}, M_{t}=175 \mathrm{GeV}, M_{Z}=91.18 \mathrm{GeV}\right)$.

Second, we collect in the set setbetaFAPT the following rules of substitutions $b_{i} \rightarrow$ $b_{i}\left(n_{f}\right)$, cf. Eq. (3),

$$
\begin{aligned}
\text { b0 }: b_{0} \rightarrow & 11-\frac{2}{3} n_{f}, \quad \text { b1 }: b_{1} \rightarrow 102-\frac{38}{3} n_{f}, \\
\text { b2 }: b_{2} \rightarrow & \frac{2857}{2}-\frac{5033}{18} n_{f}+\frac{325}{54} n_{f}^{2} \\
\text { b3 }: b_{3} \rightarrow & \frac{149753}{6}-\frac{1078361}{162} n_{f}+\frac{50065}{162} n_{f}^{2}+\frac{1093}{729} n_{f}^{3} \\
+ & {\left[3564-\frac{6508}{27} n_{f}+\frac{6472}{81} n_{f}^{2}\right] \zeta[3] . }
\end{aligned}
$$

Here we follow the same substitution strategy as in [70], but our $b_{i}$ differ from theirs $b_{i}^{\text {CKS }}$ by factors $4^{i+1}: b_{i}=4^{i+1} b_{i}^{\mathrm{CKS}}$. In parallel, the set setbetaFAPT4Pi defines substitutions $b_{i} \rightarrow b_{i}\left(n_{f}\right) /(4 \pi)$ which are more appropriate to determine coefficients $c_{i}\left(n_{f}\right)$.

\section{Appendix B. Description of the main procedures}

Here we shortly describe the main procedures of our package which can be useful for practical calculations.

- RhoBarl[L, Nf, Nu]:

general: it computes the $\ell$-loop spectral density $\bar{\rho}^{(\ell)}\left[L_{\sigma}, n_{f}, \nu\right]$;

input: the logarithmic argument $\mathrm{L}=L_{\sigma}=\ln \left[\sigma / \Lambda^{2}\right]$, the number of active flavors $\mathrm{Nf}=n_{f}$, and the power index $\mathrm{Nu}=\nu$;

output: $\bar{\rho}^{(\ell)}$;

example: In order to compute the value of the four-loop spectral density $\bar{\rho}^{(4)}[3.95,4,1.62]=0.0247209$ one has to use the command RhoBar4 [3.95, 4, 1.62].

- RhoGlob $\ell[\mathrm{L}, \mathrm{Nu}, \mathrm{Lam}]$ :

general: it computes the $\ell$-loop global spectral density $\rho^{(\ell) ; \text { glob }}\left[L_{\sigma}, \nu, \Lambda_{n_{f}=3}\right]$; 
input: the logarithmic argument $\mathrm{L}=L_{\sigma}=\ln \left[\sigma / \Lambda_{n_{f}=3}^{2}\right]$, the power index $\mathrm{Nu}=\nu$, and the QCD scale parameter $\operatorname{Lam}=\Lambda_{n_{f}=3}($ in $\mathrm{GeV})$;

output: $\rho^{(\ell) ; \text { glob; }}$

example: In order to compute the value of the four-loop spectral density $\rho^{(4) \text {;glob }}[3.95,1.62,0.350]=0.0221662$ one has to use the command RhoGlob4 [3.95, 1.62, 0.35]. 
- AcalBar $\ell[\mathrm{L}, \mathrm{Nf}, \mathrm{Nu}]$ :

general: it computes the $\ell$-loop $n_{f}$-fixed analytic coupling $\overline{\mathcal{A}}_{\nu}^{(\ell)}\left[L, n_{f}\right]$ in Euclidean domain;

input: the logarithmic argument $\mathrm{L}=\ln \left[Q^{2} / \Lambda^{2}\right]$, the number of active flavors $\mathrm{N} f=n_{f}$, and the power index $\mathrm{Nu}=\nu$;

output: $\overline{\mathcal{A}}_{\nu}^{(\ell)}$;

example: In order to compute the value of the three-loop spectral density $\overline{\mathcal{A}}_{1.62}^{(3)}[3.95,4]=0.11352$ one has to use the command AcalBar3 [3.95, 4, 1.62].

- UcalBar $\ell[\mathrm{L}, \mathrm{Nf}, \mathrm{Nu}]$ :

general: it computes the $\ell$-loop $n_{f}$-fixed analytic coupling $\overline{\mathfrak{A}}_{\nu}^{(\ell)}\left[L, n_{f}\right]$ in Minkowski domain;

input: the logarithmic argument $\mathrm{L}=\ln \left[s / \Lambda^{2}\right]$, the number of active flavors $\mathrm{Nf}=n_{f}$, and the power index $\mathrm{Nu}=\nu$;

output: $\overline{\mathfrak{A}}_{\nu}^{(\ell)}$;

example: In order to compute the value of the three-loop spectral density $\overline{\mathfrak{A}}_{1.62}^{(3)}[3.95,4]=0.1011$ one has to use the command UcalBar3 [3.95, 4, 1.62].

- AcalGlob $\ell[\mathrm{L}, \mathrm{Nu}, \mathrm{Lam}]$ :

general: it computes the $\ell$-loop global analytic coupling $\mathcal{A}_{\nu}^{(\ell) ; \text { glob }}\left[L, \nu, \Lambda_{n_{f}=3}\right]$ in Euclidean domain;

input: the logarithmic argument $\mathrm{L}=L_{\sigma}=\ln \left[\sigma / \Lambda_{n_{f}=3}^{2}\right]$, the power index $\mathrm{Nu}=\nu$, and the QCD scale parameter $\operatorname{Lam}=\Lambda_{n_{f}=3}$ (in $\left.\mathrm{GeV}\right)$;

output: $\mathcal{A}_{\nu}^{(\ell) ; \text { glob }}$;

example: In order to compute the value of the two-loop analytic coupling $\mathcal{A}_{1.62}^{(2) ; \text { glob }}[3.95,0.350]=0.103858$ one has to use the command AcalGlob2 [3.95, $1.62,0.35]$.

- UcalGlob $\ell[\mathrm{L}, \mathrm{Nu}, \mathrm{Lam}]$ :

general: it computes the $\ell$-loop global analytic coupling $\mathfrak{A}_{\nu}^{(\ell) ; \text { glob }}\left[L, \nu, \Lambda_{n_{f}=3}\right]$ in Minkowski domain;

input: the logarithmic argument $\mathrm{L}=\ln \left[s / \Lambda_{n_{f}=3}^{2}\right]$, the power index $\mathrm{Nu}=\nu$, and the QCD scale parameter $\operatorname{Lam}=\Lambda_{n_{f}=3}($ in $\mathrm{GeV})$;

output: $\mathfrak{A}_{\nu}^{(\ell) ; \text { glob }}$; 
example: In order to compute the value of the two-loop analytic coupling $\mathfrak{A}_{1.62}^{(2) \text { glob }}[3.95,0.350]=0.0932096$ one has to use the command UcalGlob2 [3.95, $1.62,0.35]$.

All $\Lambda_{n_{f}=3}$ are in GeV, all squared momentum transfer $Q^{2}$ (Euclidean), central-of-mass energy squared $s$ (Minkowski), and spectral-integration variables $\sigma$ are in $\mathrm{GeV}^{2}$. The number of loops $\ell$ is everywhere specified in the name of a procedure.

\section{References}

[1] D. V. Shirkov, I. L. Solovtsov, Analytic QCD running coupling with finite IR behaviour and universal $\bar{\alpha}_{s}(0)$ value, JINR Rapid Commun. 2[76] (1996) 5-10. arXiv: hep-ph/9604363

Analytic model for the QCD running coupling with universal $\bar{\alpha}_{s}(0)$ value, Phys. Rev. Lett. 79 (1997) 1209-1212. arXiv:hep-ph/9704333

[2] K. A. Milton, I. L. Solovtsov, Analytic perturbation theory in QCD and Schwinger's connection between the beta function and the spectral density, Phys. Rev. D55 (1997) 5295-5298. arXiv:hep-ph/9611438

[3] I. L. Solovtsov, D. V. Shirkov, Analytic approach to perturbative QCD and renormalization scheme dependence, Phys. Lett. B442 (1998) 344-348. arXiv:hep-ph/ 9711251

[4] A. P. Bakulev, S. V. Mikhailov, N. G. Stefanis, QCD analytic perturbation theory: From integer powers to any power of the running coupling, Phys. Rev. D72 (2005) 074014; Erratum: ibid. D72 (2005) 119908(E). arXiv:hep-ph/0506311

[5] A. P. Bakulev, A. I. Karanikas, N. G. Stefanis, Analyticity properties of three-point functions in QCD beyond leading order, Phys. Rev. D72 (2005) 074015. arXiv: hep-ph/0504275

[6] A. P. Bakulev, S. V. Mikhailov, N. G. Stefanis, Fractional analytic perturbation theory


D75 (2007) 056005; Erratum: ibid. D77 (2008) 079901(E). arXiv:hep-ph/0607040

[7] L. D. Landau, A. Abrikosov, L. Halatnikov, On the quantum theory of fields, Nuovo Cim. Suppl. 3 (1956) 80-104.

[8] D. J. Gross, The discovery of asymptotic freedom and the emergence of $Q C D$, Proc. Nat. Acad. Sci. 102 (2005) 9099-9108.

[9] N. N. Bogolyubov, D. V. Shirkov, Introduction to the theory of quantized fields, Intersci. Monogr. Phys. Astron. 3 (1959) 1-720.

[10] N. N. Bogolyubov, D. V. Shirkov, Introduction to the Theory of Quantum Fields, Wiley, New York, 1959, 1980. 
[11] N. N. Bogolyubov, A. A. Logunov, I. T. Todorov, Introduction to Axiomatic Quantum Field Theory, Benjamin Cummings, Reading, Massachusetts, 1975.

[12] N. N. Bogolyubov, A. A. Logunov, D. V. Shirkov, The method of dispersion relations and perturbation theory, Soviet Physics JETP 10 (1960) 574.

[13] A. V. Radyushkin, Optimized lambda-parametrization for the QCD running coupling constant in space-like and time-like regions, JINR Rapid Commun. 78 (1996) 96-99, [JINR Preprint, E2-82-159, 26 Febr. 1982]. arXiv:hep-ph/9907228

[14] N. V. Krasnikov, A. A. Pivovarov, The influence of the analytical continuation effects on the value of the QCD scale parameter $\Lambda$ extracted from the data on charmonium and upsilon hadron decays, Phys. Lett. B116 (1982) 168-170.

[15] H. F. Jones, I. L. Solovtsov, QCD running coupling constant in the timelike region, Phys. Lett. B349 (1995) 519-524. arXiv:hep-ph/9501344

[16] M. Beneke, V. M. Braun, Naive non-Abelianization and resummation of fermion bubble chains, Phys. Lett. B348 (1995) 513-520. arXiv:hep-ph/9411229

[17] P. Ball, M. Beneke, V. M. Braun, Resummation of $\left(\beta_{0} \alpha_{s}\right)^{n}$ corrections in QCD: Techniques and applications to the tau hadronic width and the heavy quark pole mass, Nucl. Phys. B452 (1995) 563-625. arXiv:hep-ph/9502300

[18] B. A. Magradze, Analytic approach to perturbative QCD, Int. J. Mod. Phys. A15 (2000) 2715-2734. arXiv:hep-ph/9911456

[19] D. S. Kourashev, The QCD observables expansion over the scheme-independent twoloop coupling constant powers, the scheme dependence reduction, hep-ph/9912410 (1999). arXiv:hep-ph/9912410

[20] B. A. Magradze, QCD coupling up to third order in standard and analytic perturbation theories, Dubna preprint E2-2000-222, 2000 [hep-ph/0010070]. arXiv: hep-ph/0010070

[21] D. S. Kourashev, B. A. Magradze, Explicit expressions for Euclidean and Minkowskian QCD observables in analytic perturbation theory, Preprint RMI-2001-18, 2001 [hep-ph/0104142] (2001). arXiv:hep-ph/0104142

[22] B. A. Magradze, Practical techniques of analytic perturbation theory of QCD, Preprint RMI-2003-55, 2003 hep-ph/0305020 (2003). arXiv:hep-ph/0305020

[23] D. S. Kourashev, B. A. Magradze, Explicit expressions for timelike and spacelike observables of quantum chromodynamics in analytic perturbation theory, Theor. Math. Phys. 135 (2003) 531-540.

[24] B. A. Magradze, A novel series solution to the renormalization group equation in QCD, Few Body Syst. 40 (2006) 71-99. arXiv:hep-ph/0512374 
[25] K. A. Milton, I. L. Solovtsov, O. P. Solovtsova, V. I. Yasnov, Renormalization scheme and higher loop stability in hadronic tau decay within analytic perturbation theory, Eur. Phys. J. C14 (2000) 495-501. arXiv: hep-ph/0003030

[26] K. A. Milton, I. L. Solovtsov, O. P. Solovtsova, Remark on the perturbative component of inclusive tau decay, Phys. Rev. D65 (2002) 076009. arXiv:hep-ph/0111197

[27] G. Cvetic, C. Valenzuela, Various versions of analytic QCD and skeleton-motivated evaluation of observables, Phys. Rev. D74 (2006) 114030. arXiv:hep-ph/0608256

[28] G. Cvetic, R. Kogerler, C. Valenzuela, Analytic QCD coupling with no power terms in UV regime, J. Phys. G37 (2010) 075001. arXiv:0912.2466

[29] B. A. Magradze, Testing the Concept of Quark-Hadron Duality with the ALEPH $\tau$ Decay Data, Few Body Syst. 48 (2010) 143-169. arXiv:1005.2674

[30] K. A. Milton, I. L. Solovtsov, O. P. Solovtsova, The Bjorken sum rule in the analytic approach to perturbative QCD, Phys. Lett. B439 (1998) 421-427. arXiv:hep-ph/ 9809510

[31] R. S. Pasechnik, D. V. Shirkov, O. V. Teryaev, Bjorken Sum Rule and $p Q C D$ frontier on the move, Phys. Rev. D78 (2008) 071902. arXiv:0808.0066

[32] K. A. Milton, I. L. Solovtsov, O. P. Solovtsova, The Gross-Llewellyn Smith sum rule in the analytic approach to perturbative QCD, Phys. Rev. D60 (1999) 016001. arXiv:hep-ph/9809513

[33] D. V. Shirkov, A. V. Zayakin, Analytic perturbation theory for practitioners and Upsilon decay, Phys. Atom. Nucl. 70 (2007) 775-783. arXiv:hep-ph/0512325

[34] N. G. Stefanis, W. Schroers, H.-C. Kim, Pion form factors with improved infrared factorization, Phys. Lett. B449 (1999) 299. arXiv:hep-ph/9807298

[35] N. G. Stefanis, W. Schroers, H.-C. Kim, Analytic coupling and Sudakov effects in exclusive processes: Pion and $\gamma^{*} \gamma \rightarrow \pi^{0}$ form factors, Eur. Phys. J. C18 (2000) 137-156. arXiv:hep-ph/0005218

[36] A. P. Bakulev, K. Passek-Kumerički, W. Schroers, N. G. Stefanis, Pion form factor in QCD: From nonlocal condensates to NLO analytic perturbation theory, Phys. Rev. D70 (2004) 033014. arXiv:hep-ph/0405062

[37] I. L. Solovtsov, D. V. Shirkov, The analytic approach in quantum chromodynamics, Theor. Math. Phys. 120 (1999) 1220-1244. arXiv:hep-ph/9909305

[38] D. V. Shirkov, Analytic perturbation theory for QCD observables, Theor. Math. Phys. 127 (2001) 409-423. arXiv: hep-ph/0012283 
[39] D. V. Shirkov, I. L. Solovtsov, Ten years of the analytic perturbation theory in QCD, Theor. Math. Phys. 150 (2007) 132-152. arXiv:hep-ph/0611229

[40] L. V. Dung, H. D. Phuoc, O. V. Tarasov, The influence of quark masses on the infrared behavior of $\alpha_{s}\left(Q^{2}\right)$ in QCD, Sov. J. Nucl. Phys. 50 (1989) 1072-1079.

[41] Y. A. Simonov, Perturbative expansions in QCD and analytic properties of $\alpha_{s}$, Phys. Atom. Nucl. 65 (2002) 135-152. arXiv:hep-ph/0109081

[42] A. V. Nesterenko, J. Papavassiliou, The massive analytic invariant charge in QCD, Phys. Rev. D71 (2005) 016009. arXiv:hep-ph/0410406

[43] A. P. Bakulev, D. V. Shirkov, Inevitability and Importance of Non-Perturbative Elements in Quantum Field Theory, in: B. Dragovich, Z. Rakic̀ (Eds.), Proceedings of the 6th MATHEMATICAL PHYSICS MEETING: Summer School and Conference on Modern Mathematical Physics, September 14-23, 2010, Belgrade, Serbia, Institute of Physics, Belgrade (Serbia), 2011, pp. 27-53. arXiv: \protect \vrulewidth0pt protect \href\{http://arxiv.org/abs/1102.2380\}\{arXiv:1102.2380\}

[44] D. V. Shirkov, A Few Lessons from pQCD Analysis at Low Energies, arXiv:1202.3220 [hep-ph] (2012). arXiv:1202.3220

[45] A. I. Karanikas, N. G. Stefanis, Analyticity and power corrections in hard-scattering hadronic functions, Phys. Lett. B504 (2001) 225-234; Erratum: ibid. B636 (2006) 330. arXiv:hep-ph/0101031

[46] N. G. Stefanis, Perturbative logarithms and power corrections in QCD hadronic functions: A unifying approach, Lect. Notes Phys. 616 (2003) 153-166. arXiv: hep-ph/0203103

[47] A. P. Bakulev, Global Fractional Analytic Perturbation Theory in QCD with Selected Applications, Phys. Part. Nucl. 40 (2009) 715-756. arXiv:0805.0829[hep-ph]

[48] G. Cvetic, A. V. Kotikov, Analogs of noninteger powers in general analytic QCD, J. Phys. G39 (2012) 065005. arXiv:1106.4275

[49] D. J. Broadhurst, A. L. Kataev, C. J. Maxwell, Renormalons and multiloop estimates in scalar correlators, Higgs decay and quark-mass sum rule, Nucl. Phys. B592 (2001) 247-293. arXiv:hep-ph/0007152

[50] A. P. Bakulev, S. V. Mikhailov, N. G. Stefanis, Higher-order QCD perturbation theory in different schemes: From FOPT to CIPT to FAPT, JHEP 1006 (2010) 085 (1-38). arXiv:1004.4125

[51] R. S. Pasechnik, D. V. Shirkov, O. V. Teryaev, O. P. Solovtsova, V. L. Khandramai, Nucleon spin structure and pQCD frontier on the move, Phys. Rev. D81 (2010) 016010. arXiv:0911.3297 
[52] G. Cvetic, A. Y. Illarionov, B. A. Kniehl, A. V. Kotikov, Small-x behavior of the structure function $F_{2}$ and its slope $\partial \ln \left(F_{2}\right) / \partial \ln (1 / x)$ for 'frozen' and analytic strongcoupling constants, Phys. Lett. B679 (2009) 350-354. arXiv:0906.1925

[53] A. V. Kotikov, V. G. Krivokhizhin, B. G. Shaikhatdenov, Analytic and 'frozen' $Q C D$ coupling constants up to NNLO from DIS data, Phys. Atom. Nucl. 75 (2012) 507-524. arXiv: 1008.0545

[54] A. V. Nesterenko, C. Simolo, QCDMAPT: program package for Analytic approach to QCD, Comput. Phys. Commun. 181 (2010) 1769-1775. arXiv:1001.0901

[55] A. Nesterenko, C. Simolo, QCDMAPT_F: Fortran version of QCDMAPT package, Comput. Phys. Commun. 182 (2011) 2303-2304. arXiv:1107.1045

[56] S. Wolfram, Mathematica - a system for doing mathematics by computer, AddisonWesley, New York, 1988.

[57] D. J. Gross, F. Wilczek, Ultraviolet behavior of nonabelian gauge theories, Phys. Rev. Lett. 30 (1973) 1343-1346.

[58] D. J. Gross, F. Wilczek, Asymptotically free gauge theories. 1, Phys. Rev. D8 (1973) 3633-3652.

[59] H. D. Politzer, Reliable perturbative results for strong interactions?, Phys. Rev. Lett. 30 (1973) 1346-1349.

[60] D. R. T. Jones, Two-Loop Diagrams in Yang-Mills Theory, Nucl. Phys. B75 (1974) 531. doi:10.1016/0550-3213(74)90093-5

[61] W. E. Caswell, Asymptotic Behavior of Non-Abelian Gauge Theories to Two-Loop Order, Phys. Rev. Lett. 33 (1974) 244. doi:10.1103/PhysRevLett.33.244

[62] E. Egorian, O. V. Tarasov, Two-loop renormalization of the QCD in an arbitrary gauge, Theor. Math. Phys. 41 (1979) 863- 869.

[63] O. V. Tarasov, A. A. Vladimirov, A. Y. Zharkov, The Gell-Mann-Low Function of QCD in the Three-Loop Approximation, Phys. Lett. B93 (1980) 429-432. doi: 10.1016/0370-2693(80)90358-5

[64] S. A. Larin, J. A. M. Vermaseren, The three-loop QCD beta function and anomalous dimensions, Phys. Lett. B303 (1993) 334-336. arXiv:hep-ph/9302208 doi: 10.1016/0370-2693(93)91441-0

[65] T. van Ritbergen, J. A. M. Vermaseren, S. A. Larin, The four-loop beta function in quantum chromodynamics, Phys. Lett. B400 (1997) 379-384. arXiv:hep-ph/ 9701390 
[66] M. Czakon, The Four-loop QCD beta-function and anomalous dimensions, Nucl. Phys. B710 (2005) 485-498. arXiv:hep-ph/0411261

[67] B. A. Magradze, The gluon propagator in analytic perturbation theory, in: F. L. Bezrukov, V. A. Matveev, V. A. Rubakov, A. N. Tavkhelidze, S. V. Troitsky (Eds.), Proceedings of the 10th International Seminar Quarks'98, Suzdal, Russia, 18-24 May 1998, INR RAS, Moscow, 1999, pp. 158-171. arXiv:hep-ph/9808247

[68] E. Gardi, G. Grunberg, M. Karliner, Can the QCD running coupling have a causal analyticity structure?, JHEP 07 (1998) 007. arXiv:hep-ph/9806462

[69] A. V. Garkusha, A. L. Kataev, The absence of QCD $\beta$-function factorization property of the generalized Crewther relation in the 't Hooft $\bar{M} S$-based scheme, Phys. Lett. B705 (2011) 400-404. arXiv:1108.5909

[70] K. G. Chetyrkin, J. H. Kuhn, M. Steinhauser, RunDec: A Mathematica package for running and decoupling of the strong coupling and quark masses, Comput. Phys. Commun. 133 (2000) 43-65. arXiv:hep-ph/0004189 doi:10.1016/S0010-4655(00) 00155-7 